\title{
„Minimal-invasiv an Ihrer Seite“: Interventionsradiologie startet Informationsoffensive
}

Die Interventionelle Radiologie verfügt über ein breites Spektrum in Diagnose und Therapie - von der Gewebeentnahme über die Therapie der Schaufensterkrankheit bis hin zur Schlaganfallbehandlung. Nicht alle überweisenden Ärztinnen und Ärzte wissen jedoch um dieses umfangreiche Angebot. Die Deutsche Gesellschaft für Interventionelle Radiologie und minimal-invasive Therapie (DeGIR) hat daher eine Informationsbroschüre entwickelt, die Interventionsradiologinnen und -radiologen ab sofort zur Information ihrer zuweisenden Kolleginnen und Kollegen nutzen können.

In kompakter Form bietet die Publikation einen Überblick über die große Bandbreite der Interventionsradiologie. Anschaulich werden die Therapieoptionen anhand der häufigsten Erkrankungsbilder geschildert. Zahlreiche Abbildungen erlauben einen Einblick „hinter die Kulissen“ und visualisieren die minimal-invasiven Eingriffe; kurze Fallbeschreibungen erleichtern den Einstieg.

\section{Hochwertiges in der Hand: Die Printausgabe}

Die Broschüre gibt es in zwei Varianten: Als interaktive Digitalausgabe oder als hochwertiges Printprodukt. Mit der gedruckten Broschüre überzeugen Sie besonders im persönlichen Kontakt bei hausärztlichen Kolleginnen und Kollegen. Egal, ob beim ersten Kontakt oder im Rahmen einer langjährigen Zusammenarbeit - auch erfahrene Zuweiserinnen und Zuweiser lernen sicher noch neue Einsatzmöglichkeiten minimalinvasiver Therapien kennen. Mit dem Praxis- oder Klinikstempel auf der Rückseite lässt sich jedes Exemplar individualisieren. Selbstverständlich kann die Broschüre auch an interessierte Patientinnen und Patienten weitergegeben werden.

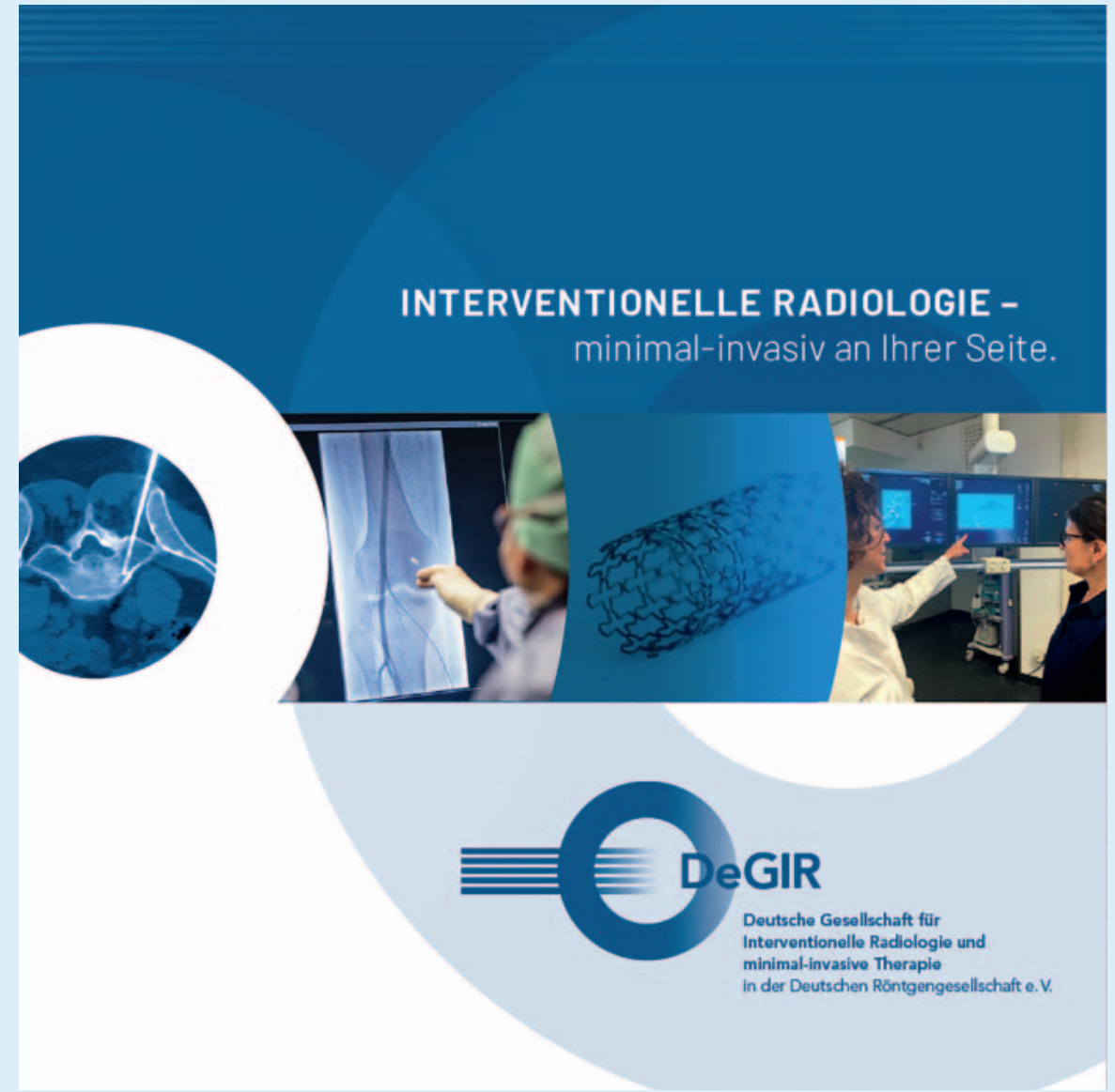

Interaktiv für die digitale Kommunikation:

\section{Die Webausgabe}

Die digitale Version überzeugt durch ein informatives Video, das auf lebendige Art und Weise in die Welt der Interventionsradiologie einführt. Ein interaktiver 360-GradRundgang durch die Angiografie und großformatige Bildergalerien sorgen für zusätzlichen Informationsgehalt. Digital lässt sich die Broschüre beispielsweise nutzen, um in der E-Mail-Signatur auf interventionsradiologische Leistungen hinzuweisen. Den Link zur Digitalausgabe finden Sie auf www.degir.de.
Jetzt bestellen: Ihre Informationsbroschüren zur Interventionsradiologie!

Ihre Printexemplare mit Platz für Ihren Praxis- oder Klinikstempel erhalten Sie kostenfrei* in Ihrer DeGIR-Geschäftsstelle:

DeGIR-Geschäftsstelle

c/o Deutsche Röntgengesellschaft e. V.

Ernst-Reuter-Platz 10

10587 Berlin

Telefon: 030 916 070-0/Fax: -22

E-Mail: degir@drg.de

Internet: www.degir.de 
DIE THEMEN IM ŨBERBLICK.

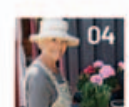

T. W06 "Endlich eine eindeutige Diagnose.* $\frac{10=100}{2}$
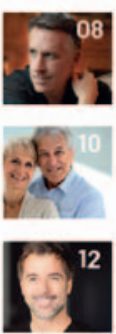

Beim Wettlauf mit meinen Enkeln bin ich jetzt wieder dabeil Arterielle Verschilusskrankheit (pAVK) Bildgesteuerte Biopsien

Meine interventionelle Radiologin hat mich von meinem Lebertumor geheilt. Behandlung von Lebertumoren

\$Mein Mann kann wieder durchschlafen.s Myomembolisationund Prost ataembolisation

Jetzt feiere ich zweime im Jahr Ge burtstag! e

Sehlagantallintrakranielle Thrombektomis Sehlagantallintrakrande
und Karotisstenose

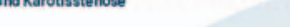

BUNDESWEIT OPTIMAL VERSORGT.

In Deutschland ist uberall ein Spezialist für minimalinvasive Eingriffe in Ihrer Nathe. Soist eine flächendeckende Versorgung gewahrieistet.

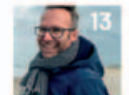

Fine kleine Metallspirale war mein Lebensretter!

Nottallbehandung durch Embolisation

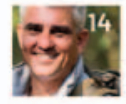

Einzweites Leben

wurde mir geschenkt. s

Behandlung des Aortenaneurysma

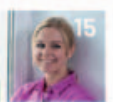

Einkleiner Eingriff hat meinen

Rückenschmerzen ein Ende gemacht,

Bildgesteverte Schmerztherapien

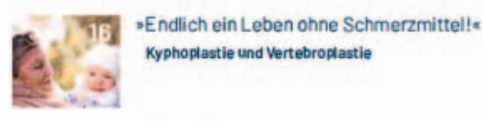

-Endlich ein Leben ohne Schmerzmitte!!

造 id

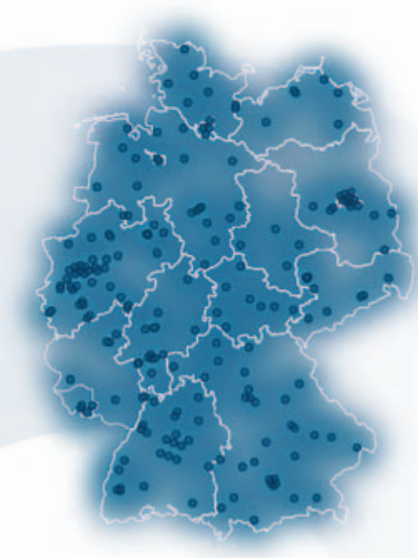

ARTERIELLE VERSCHLUSSKRANKHEIT
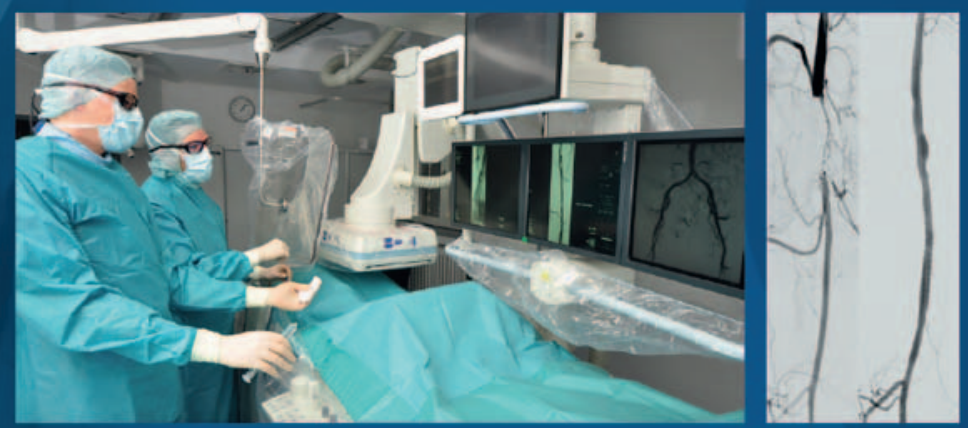

Arterielle

\section{Verschlusskrankheit}

(pAVK)

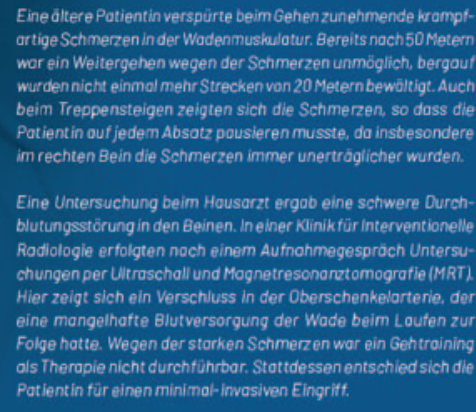

Eine ätere Patientin verspürte beim Gehen zunehmende krampt wurdennicht einmol mehrStrechen van 20 Metern bew bltigt. Auch beim Treppensteigen zeigten sich die Schmerzen, so dass die rer

Eine Untersuchung beim Housarzt ergob eine schwere Durchblutungsstorung in den Beinen. In einer Ninikfü Interventionelle Rodiclogle erfolgten noch einem Au fnohmegesproch Untersu-

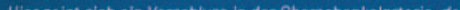
eine mangelhafte Blutversorgung der Wade beim Laufen zur Patientin für ainen minimal-invasiven Eingrift 

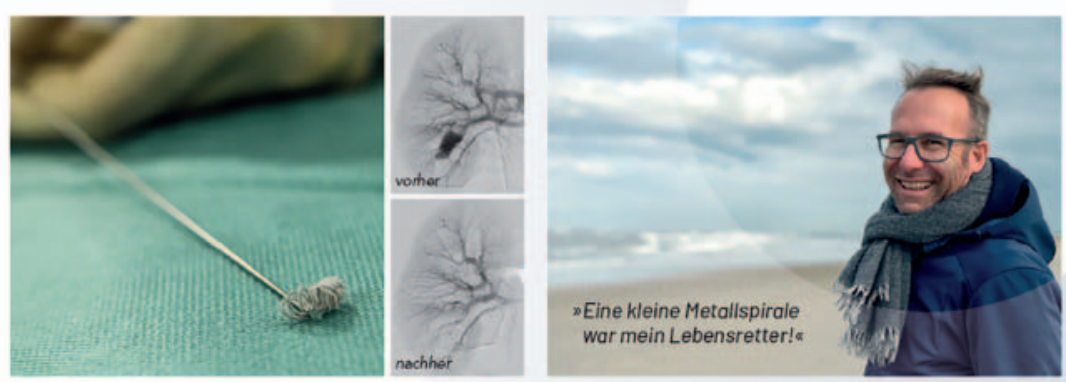

\section{Notfallbehandlung}

durch Embolisation

EinPatient leidet seitüberzehn Jahrenunter Nierensteinen. Einige be sonders hartnäckige mussten minimal-invasiv entfernt werden. Wenige Tage nach dem erfolgreichen Eingriff kam es zustorken Schmerzen und blutigem Urin

\section{ANEURYSMEN}

Blutungen aus Arterien (Schlagadern) sind oft lebensbedrohlich. Sie kōnnen verschiedene Ursachen haben. Dazu gehören Verletzungen wie im Falle des Nierensteinpatienten oder nach Unf Oie urologische Untersuchung ergab, doss ein krattiges Blutgefä beim Entzûndungen oder Gefäbaussackungen (Aneurysmen) bei ArterioEingriff verletzt worden wor. Dies hatte einen Blutvertust in đas Nieren- sklerose. Magen-/Darmgeschwüren oder Tumoren. Im Gehirn können Eingrift verletzt worden wor. Oies hatte einen Blutvertust in das Nieren- sklerose. Magen-/Oarmgeschwüren oder Tumoren. Im Gehir
becken und in die Blose zur Folge.
Aneurysmen angeboren sein und lebensgetährlich bluten.

Es erfolgte eine sofortige Verlegung van der intensivstotion in die in- $0 \mathrm{~b}$ eine Operation oder eine radiologische Intervention die richtige terventionelle Rodiologie. Hiler wurde über die Leistenorterle ein Fun- Behandlung ist, wirdimmer fachubergreifend entschieden. Oft wird in rungskatheter mit einem dūnnen Mikrokotheter von der Leiste bis in die Notfallsituationen ein minimal-invasiver Eingritt gewàht: Anders ats Nierenorterie geführt. Das Ende des Mikrokotheters wurde unmittelibar bei einer Operationkann der interventionelle Radiologe nàmlichmittels vor der Gefäbverletzung platziert, um danüber Spiralen (Coils) einzuführen. Rönt gen-Bildgebung, Katheterund Kontrastmittel den Ort der Blutung Die Colis bestehen aus Plotin und rollen sich in einem fest definierten sehr prïzise finden und punktgenau ther apieren. Die interventionelle Durchmesser ein, der an die GröBe des BlutgeföBes angeposst ist. Wenn Behandlung mit Metallspiralen oder Spezialklebern stoppt die Blutung

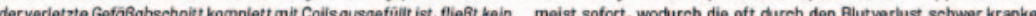
dert mehr hinein. Somit waren die lebensgetihn liche Blutung gebonnt Pationten schnell wieder in und eine drohende Entternung der Niere verhindert. 